\title{
Shape Representation and Indexing Based on Region Connection Calculus and Oriented Matroid Theory
}

\author{
Ernesto Staffetti ${ }^{1}$, Antoni Grau ${ }^{2}$, \\ Francesc Serratosa ${ }^{3}$, and Alberto Sanfeliu ${ }^{1}$ \\ 1 Institute of Industrial Robotics (CSIC-UPC) \\ Llorens i Artigas 4-6, 08028 Barcelona Spain \\ \{estaffetti, asanfeliu\}@iri.upc.es \\ 2 Department of Automatic Control, Technical University of Catalonia \\ Pau Gargallo 5, 08028 Barcelona Spain \\ antoni.grau@upc.es \\ 3 Department of Computer Engineering and Mathematics \\ Rovira i Virgili University, \\ Av. Paisos Catalanes 26, 43007 Tarragona Spain \\ francesc.serratosa@etse.urv.es
}

\begin{abstract}
In this paper a novel method for indexing views of 3D objects is presented. The topological properties of the regions of the views of a set of objects are used to define an index based on the region connection calculus and oriented matroid theory. Both are formalisms for qualitative spatial representation and reasoning and are complementary in the sense that whereas the region connection calculus encodes information about connectivity of pairs of connected regions of the view, oriented matroids encode relative position of the disjoint regions of the view and give local and global topological information about their spatial distribution. This indexing technique is applied to 3D object hypothesis generation from single views to reduce candidates in object recognition processes.
\end{abstract}

\section{Introduction}

In this paper we present a new method for indexing views of $3 \mathrm{D}$ objects which is applied to 3D object hypothesis generation from single views to reduce candidates in $3 \mathrm{D}$ object recognition processes.

Given a set of views of different 3D objects, the problem of object recognition using a single view becomes the problem of finding a subset of the set of regions in the image with a relational structure identical to that of a member of the set of views. The standard way to reduce the complexity of shape matching is subdividing the problem into a hypothesis generation followed by a verification. To be of interest for object recognition, hypothesis generation should be a relatively fast although imprecise procedure in which several possible candidates for matching are generated. In this way the verification can be carried out using a more complex, and therefore, slower procedure [1] over a reduced number of 


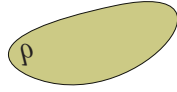

or

$D C\left(\alpha^{2} \rho\right)$

$(9)$

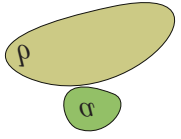

$E C\left(\alpha^{2} \rho\right)$

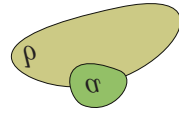

$\mathrm{bO}\left(\alpha^{2} \rho\right)$

(c)

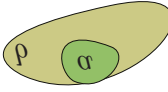

$\operatorname{Lbb}\left(a^{2} \rho\right)$

(q)

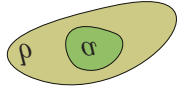

$\eta \perp b b\left(a^{2} \rho\right)$

(G)

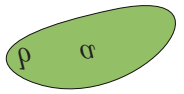

$E \sigma\left(\alpha^{2} \rho\right)$

(t)

Fig. 1. Some of the 8 possible relative positions of two regions and the corresponding descriptions using the formalism of the region connection calculus. The other two can be obtained from $(d)$ and $(e)$ interchanging $a$ with $b$. In situation (a) $a$ is disconnected from $b$, in (b) $a$ is externally connected to $b$, in situation $(c) a$ is partially overlapped to $b$, in (d) $a$ is tangential proper part of $b$, in (e) $a$ is non-tangential proper part of $b$ and, finally, in situation (f) $a$ and $b$ coincide.

candidates. The hypothesis generation can be carried out very efficiently if it is formulated as an indexing problem where the set of views of the set of 3D objects are stored into a table that is indexed by some function of the views themselves.

In this paper an indexing technique that combines the region connection calculus and oriented matroid theory is presented. More precisely, the type of connectivity between connected regions of the views is described by means of the formalism of the region connection calculus [2], whereas the topological properties of the disconnected regions of the views are encoded into a data structure called set of cocircuits [3]. The set of cocircuits, that are one of the several combinatorial data structure referred to as oriented matroids, encode incidence relations and relative position of the elements of the image and give local and global topological information about their spatial distribution. Reasoning with the region connection calculus is based on composition tables, while oriented matroids permit algebraic techniques to be used. These two descriptions merged are used as an index of the database.

This indexing method is employed to the hypothesis generation for 3D object recognition from single views that can be regarded as a qualitative counterpart of the geometric hashing technique [4]. For another approach to shape representation and indexing based on combinatorial geometry see [5].

The region connection calculus and oriented matroids are introduced in Section 2 whereas Section 3 describes the proposed indexing method. In Section 4 some experimental results are reported and Section 5 contains the conclusions.

\section{Qualitative Spatial Representation}

Qualitative reasoning is based on comparative knowledge rather than on metric information. Many methods for shape representation and analysis are based on extracting points and edges which are used to define projectively invariant descriptors. In this paper, instead of points, regions of the images are taken into account. The motivation behind this choice is that the regions of an image can be more reliably extracted than vertices and edges. In the following sections two formalisms for qualitative representation and reasoning are described: the first 


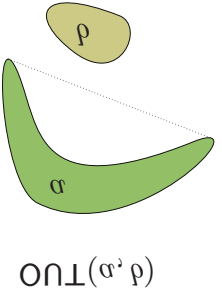

$(\alpha)$

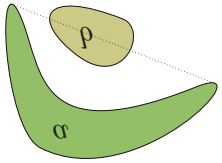

b-Іиट $\left(a^{2} \rho\right)$

$(\rho)$

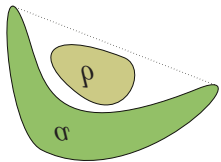

Іиг $\left(\alpha^{2} \rho\right)$

(c)

Fig. 2. Some of the possible positions of a convex region with respect to the convex hull of a non-convex one.

one is based on the region connection calculus and the second one is derived from oriented matroid theory.

\subsection{Region Connection Calculus}

For spatially extended objects we can qualitatively distinguish the interior, the boundary, and the exterior of the object, without taking into account the concrete shape or size of the object.

A set theoretical analysis of the possible relations between objects based on the above partition is provided by [6]. The relation between objects that they examine is the intersection between their boundaries and interiors. This setting is based on the distinction of the values empty and non-empty for the intersection.

Some variants of this theory were developed by Cohn and his coworkers in a series of papers (see for example [2]). In this work the distinction between interior and the boundary of an object is abandoned, and eight topological relations derived from the single binary relation "connected to" are taken into account. Some of them are represented in Fig. 1. Some of these relations, namely those of Fig. 1.d and Fig. 1.e, are not symmetrical and, following the notation of [2], their inverses are denoted $\operatorname{TPPi}(a, b)$ and $\operatorname{NTTPi}(a, b)$, respectively.

Furthermore in [2] the theory is extended to handle concave objects by distinguishing the regions inside and outside of the convex hull of the objects. A convex object can be inside, partially inside or outside the convex hull of a non-convex one (Fig. 2). If both regions are non-convex 23 relations between them can be defined. These relations permit qualitative description of rather complex relations, such as that represented in Fig. 3. Moreover, by means of this formalism called region connection calculus it is possible, for instance, to infer the relative position of two regions knowing their position with respect to a third one. Reasoning with the region connection calculus is essentially based on composition tables.

\subsection{Oriented Matroids}

Oriented matroid theory [3], [7], [8] is a broad setting in which the combinatorial properties of geometrical configurations can be described and analyzed. It 


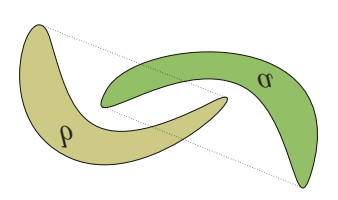

Fig. 3. With the formalism of the region connection calculus the relation between these two disconnected non-convex regions, where $a$ is partially inside the convex hull of $b$ and vice versa, is denoted by P-INS_P-INSi_DC $(a, b)$.

provides a common generalization of a large number of different mathematical objects usually treated at the level of usual coordinates. In this section oriented matroids will be introduced over arrangements of points using two combinatorial data structures called chirotope and set of cocircuits, which represent the main tools to translate geometric problems into this formalism. In the abstraction process from the concrete configuration of points to the oriented matroid, metric information is lost but the structural properties of the configuration of points are represented at a purely combinatorial level.

Oriented Matroids of Arrangements of Points. Given a point configuration in $\mathbb{R}^{d-1}$ whose elements are the columns of the matrix $\mathbf{P}=\left(p_{1}, p_{2}, \ldots, p_{n}\right)$, the associated vector configuration is a finite spanning sequence of vectors $\left\{x_{1}, x_{2}\right.$, $\left.\ldots, x_{n}\right\}$ in $\mathbb{R}^{d}$ represented as columns of the matrix $X=\left(x_{1}, x_{2}, \ldots, x_{n}\right)$ where each point $p_{i}$ is represented in homogeneous coordinates as $x_{i}=\left(\begin{array}{c}p_{i} \\ 1\end{array}\right)$.

To encode the combinatorial properties of the point configuration we can use a data structure called chirotope [8], which can be computed by means of the associated vector configuration $X$. The chirotope of $X$ is the map

$$
\begin{aligned}
\chi_{X}: & \{1,2, \ldots, n\}^{d} \rightarrow\{+, 0,-\} \\
& \left(\lambda_{1}, \lambda_{2}, \ldots, \lambda_{d}\right) \mapsto \operatorname{sign}\left(\left[x_{\lambda_{1}}, x_{\lambda_{2}}, \ldots, x_{\lambda_{d}}\right]\right)
\end{aligned}
$$

that assigns to each $d$-tuple of vectors of the finite configuration $X$ a sign + or - depending on whether it forms a basis of $\mathbb{R}^{d}$ having positive or negative orientation, respectively. This function assigns the value 0 to those $d$-tuples that do not constitute a basis of $\mathbb{R}^{d}$. The chirotope describes the incidence structure between the points of $X$ and the hyperplanes spanned by the same points and, at the same time, encodes the relative position of the points of the configuration with respect to the hyperplanes that they span.

Consider the point configuration $P$ represented in Fig. 4 whose associated vector configuration $X$ is given in Table 1 .

Table 1. Vector configuration that corresponds to the planar point configuration represented in Fig. 4.

$$
\begin{array}{|c|c|c|}
\hline x_{1}=(0,3,1)^{T} & x_{2}=(-3,1,1)^{T} & x_{3}=(-2,-2,1)^{T} \\
\hline x_{4}=(2,-2,1)^{T} & x_{5}=(3,1,1)^{T} & x_{6}=(0,0,1)^{T} \\
\hline
\end{array}
$$




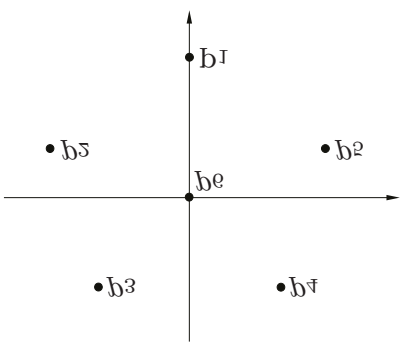

Fig. 4. A planar point configuration.

Table 2. Chirotope of the planar point configuration represented in Fig. 4.

\begin{tabular}{|l|l|l|l|l|}
\hline$\chi(1,2,3)=+$ & $\chi(1,2,4)=+$ & $\chi(1,2,5)=+$ & $\chi(1,2,6)=+$ & $\chi(1,3,4)=+$ \\
\hline$\chi(1,3,5)=+$ & $\chi(1,3,6)=+$ & $\chi(1,4,5)=+$ & $\chi(1,4,6)=-$ & $\chi(1,5,6)=-$ \\
\hline$\chi(2,3,4)=+$ & $\chi(2,3,5)=+$ & $\chi(2,3,6)=+$ & $\chi(2,4,5)=+$ & $\chi(2,4,6)=+$ \\
\hline$\chi(2,5,6)=-$ & $\chi(3,4,5)=+$ & $\chi(3,4,6)=+$ & $\chi(3,5,6)=+$ & $\chi(4,5,6)=+$ \\
\hline
\end{tabular}

Table 3. Set of cocircuits of the planar point configuration represented in Fig. 4.

\begin{tabular}{|l|l|l|}
\hline$(0,0,+,+,+,+)$ & $(0,-, 0,+,+,+)$ & $(0,-,-, 0,+,-)$ \\
\hline$(0,-,-,-, 0,-)$ & $(0,-,-,+,+, 0)$ & $(+, 0,0,+,+,+)$ \\
\hline$(+, 0,-, 0,+,+)$ & $(+, 0,-,-, 0,-)$ & $(+, 0,-,-,+, 0)$ \\
\hline$(+,+, 0,0,+,+)$ & $(+,+, 0,-, 0,+)$ & $(+,+, 0,-,-, 0)$ \\
\hline$(+,+,+, 0,0,+)$ & $(-,+,+, 0,-, 0)$ & $(-,-,+,+, 0,0)$ \\
\hline
\end{tabular}

The chirotope $\chi_{X}$ of this vector configuration is given by the orientations listed in Table 2 . The element $\chi(1,2,3)=+$ indicates that in the triangle formed by $p_{1}, p_{2}$, and $p_{3}$ these points are counterclockwise ordered. These orientations can be rearranged in an equivalent data structure called set of cocircuits of $X$ shown in Table 3. In this planar case, the set of cocircuits of $X$ is the set of all partitions generated by the lines passing through two points of the configuration. For example, $(0,0,+,+,+,+)$ means that the points $p_{3}, p_{4}, p_{5}$, and $p_{6}$ lie on the half plane determined by the line through the points $p_{1}$ and $p_{2}$. Reversing all the signs of the set of cocircuits we obtain an equivalent description of the planar arrangement of points.

Besides chirotopes and cocircuits there are several data structures capable of encoding the topological properties of a point configuration. In [8] their definitions can be found and it is shown that all of them are equivalent and are referred to as oriented matroids.

Oriented Matroid of Arrangements of Regions. Consider a segmented view of a $3 \mathrm{D}$ object. Extracting the oriented matroid of a view is not straightforward since the regions that form the image cannot be reduced to points, taking for instance their centroids, without losing essential topological information for 
object recognition. Therefore, the convex hull [9] of each region is employed to represent the region itself. Then, pairs of the resulting convex polygons are considered and the oriented matroid is computed based on the spatial location of the other convex regions of the image with respect to the two lines arising in merging the convex hulls of pairs disconnected regions.

Consider, for instance, the ordered pair of convex regions $(S, T)$ of Fig. 5.a. It is easy to see that the convex hull of these two planar convex disconnected polygonal regions is a polygon whose set of vertices is included in the union of the set of vertices of $S$ and $T$. On the contrary, the set of edges of the convex hull of $S$ and $T$ is not included in the union of their set of edges. Indeed, two new "bridging edges," $e_{1}$ and $e_{2}$, appear as illustrated in Fig. 5.a. Actually, efficient algorithms for merging convex hulls are based on finding these two edges [10].

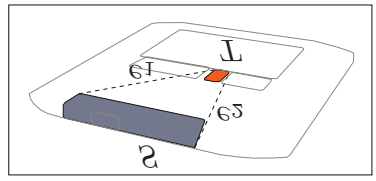

$(\propto)$

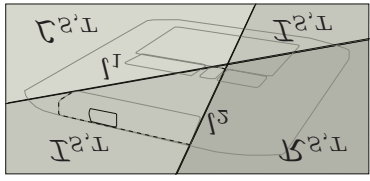

$(\rho)$

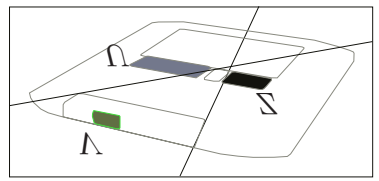

(c)

Fig. 5. Steps of encoding of the combinatorial properties of a view of an object into a chirotope.

Consider the two lines $l_{1}$ and $l_{2}$ that support $e_{1}$ and $e_{2}$. These two lines divide the image into three or four zones depending on the location of their intersection point with respect to the image. Let $\mathcal{R}_{S, T}, \mathcal{L}_{S, T}$ (Fig. 5.b) be, respectively, the rightmost and leftmost zones with respect to $l_{1}$ and $l_{2}$ and $\mathcal{I}_{S, T}$ the zone of the image comprised between them.

Since, $\mathcal{R}_{S, T}, \mathcal{L}_{S, T}$ and $\mathcal{I}_{S, T}$ can be univocally determined from the ordered couple of region $(S, T)$, the location of a region $U$ with respect to the regions $(S, T)$ of the image is encoded into a chirotope using the following rule

$$
\chi(S, T, U)= \begin{cases}+ & \text { if } U \in \mathcal{L}_{S, T}, \\ 0 & \text { if } U \in \mathcal{I}_{S, T}, \\ - & \text { if } U \in \mathcal{R}_{S, T} .\end{cases}
$$

It has been implicitly assumed that $U$ is completely contained into either $\mathcal{R}_{S, T} \mathcal{L}_{S, T}$ or $\mathcal{I}_{S, T}$ but, in general, it belongs to more that one of them. In this case, since the ratio of areas is an affine invariant, introducing an approximation, we can choose the sign based on which region contains the largest portion of the area of $U$. For instance, if regions $U, V$ and $Z$ are located as in Fig. 5.c we have that $\chi(S, T, U)=+, \chi(S, T, V)=0$ and $\chi(S, T, Z)=-$.

\subsection{Invariance of the Representation}

Consider a 3D point configuration and one of its views. The combinatorial structure of the $3 \mathrm{D}$ point configuration and that of its $2 \mathrm{D}$ perspective projection are 
related in the following way: if $x_{0}$ represents in homogeneous coordinates the center of the camera, $p_{0}$, we have that

$$
\operatorname{sign}\left[\bar{x}_{i}, \bar{x}_{j}, \bar{x}_{k}\right]=\operatorname{sign}\left[x_{i}, x_{j}, x_{k}, x_{0}\right]
$$

where $x_{i}, x_{j}$ and $x_{k}$ are the homogeneous coordinates of the $3 \mathrm{D}$ points $p_{i}, p_{j}$ and $p_{k}$, and $\bar{x}_{i}, \bar{x}_{j}$ and $\bar{x}_{k}$ are those of the corresponding points in the view, $\bar{p}_{i}$, $\bar{p}_{j}$ and $\bar{p}_{k}$. Equation (1) can be regarded as a projection equation for chirotopes.

It is easy to see that, whereas the matrix that represents in homogeneous coordinates the vertices of a projected set of points is coordinate-dependent, an oriented matroid is a coordinate-free representation. Moreover, the representation of object views based on oriented matroid is a topological invariant, that is, an invariant under homeomorphisms. Roughly speaking, this means that the oriented matroid that represents the arrangement of points of a view of an object does not change when the points undergo a continuous transformation that does not change any orientation of the chirotope. Doe to this property this representation is robust to discretization errors of the image as well as to small changes of the point of view that does not change any orientation of the chirotope.

Since projective transformations can be regarded as special homeomorphisms, we can assert that the representation of the projected set of points based on oriented matroids is projective invariant. However, since affine and Euclidean transformations are special projective transformations, the oriented matroid of the projected set of points of a view of an object does not change under rotations, translations, and affine transformations of the planar arrangement of points themselves.

These considerations can be extended to the case in which oriented matroids represent arrangements of planar regions. Since the ratio of areas is not invariant under projective transformations this representation will be invariant only under affine and Euclidean transformations of the views.

\section{Indexing Views of 3D Objects}

The process of indexing a database of views of a set of objects starts with some preliminary choices, namely the features used to characterize the regions of the segmented views of the set of 3D objects. Suppose that hue and area are used to characterize each region.

Another parameter to choose is the number of levels in which the hue is quantized and the number of regions having the same hue that will be taken into account. These choices, of course, depend on the properties of the views of the database.

Then, the views are segmented according to these choices and the convex hull of each region is computed. As a consequence, the resulting images are compositions of convex polygonal regions that can be disconnected or partially or completely overlapped. In Fig. 6 are represented two views of two objects in which a hue quantization with 6 levels $W, R, Y, G, B$ and $N$ has been applied and only the two biggest regions with the same hue value are taken into account. 
Let $(W, R, Y, G, B, N)$ be the ordered tuple of hue levels considered. For example, labels $G_{1}$ and $G_{2}$ in Fig. 6 denote, respectively, the first and the second regions of the views with the biggest area having the same hue value $G$.

The type of connection between the existing regions is described using the formalism of the region connection calculus. For each pair of disconnected regions the set of cocircuits is computed. This is done for each view of the database and this information is combined into a unique index table whose entries are spatial combinations of features and whose records contain a list of the views in which each combination is present.

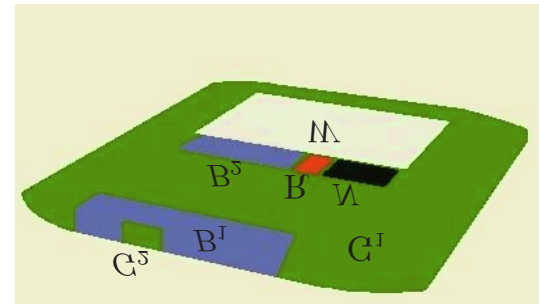

Object 1

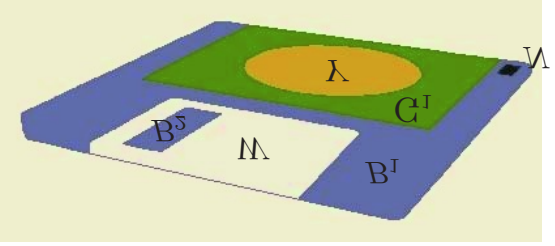

Object 2

Fig. 6. Two views of two objects whose topological properties are indexed in Table 4.

In Table 4 the index of the topological properties of the two views $v_{1,1}$ and $v_{1,2}$ of the objects represented in Fig. 6 is reported.

In the first column the relation between ordered couples of regions is described in terms of the region connection calculus. The symbol " $\emptyset$ " for a certain couple $(S, T)$ indicates that no view contains two regions having features $S$ and $T$. This is the case of the regions $R$ and $Y$.

When $S$ and $T$ are disconnected, the corresponding cocircuit is present in the index. The symbol "*" in correspondence with a certain feature indicates that no region with that feature is present in the views listed in the record. For example, the cocircuit $W R$ contains a $*$ in the column $Y$ because no region with the $Y$ feature is present in $v_{1,1}$.

If $(S, T)$ is a couple of connected regions, the corresponding row of the index is empty because the cocircuit cannot be computed.

\subsection{Hypothesis Generation for Object Recognition}

Given a database of views of a set of $3 \mathrm{D}$ objects and a view $v_{i}$ of one of them, not necessarily contained in the database, its set of cocircuits is computed. Each cocircuit is used to access the table that constitutes the index of the database.

Then the views that best match $v_{i}$ are selected based on the number of correspondences they have with $v_{i}$ in terms of cocircuits.

It is easy to see that this method for hypothesis generation, that can be regarded as a qualitative version of the geometric hashing technique [4], is also robust to partial occlusions of the objects. Indeed, if a region of an image is 
Table 4. Index of the topological properties of the two views $v_{1,1}$ and $v_{1,2}$ of the two objects represented in Fig. 6.

\begin{tabular}{|l|c|c|c|c|c|c|c|c|c|c|}
\hline & Connection & $\mathrm{W}$ & $\mathrm{R}$ & $\mathrm{Y}$ & $G_{1}$ & $G_{2}$ & $B_{1}$ & $B_{2}$ & $\mathrm{~N}$ & Objects \\
\hline$W R$ & $\mathrm{DC}$ & 0 & 0 & $*$ & 0 & 0 & 0 & - & + & $v_{1,1}$ \\
\hline$W Y$ & $\mathrm{DC}$ & 0 & $*$ & 0 & 0 & $*$ & 0 & 0 & - & $v_{1,2}$ \\
\hline$W G_{1}$ & $\mathrm{NTPP}$ & & & & & & & & & $v_{1,1}$ \\
\hline$W G_{1}$ & $\mathrm{DC}$ & 0 & $*$ & 0 & 0 & $*$ & 0 & 0 & 0 & $v_{1,2}$ \\
\hline$W G_{2}$ & $\mathrm{DC}$ & 0 & 0 & $*$ & 0 & 0 & + & 0 & 0 & $v_{1,1}$ \\
\hline$W B_{1}$ & $\mathrm{DC}$ & 0 & 0 & $*$ & 0 & 0 & 0 & 0 & 0 & $v_{1,1}$ \\
\hline$W B_{1}$ & $\mathrm{NTPP}$ & & & & & & & & & $v_{1,2}$ \\
\hline$W B_{2}$ & $\mathrm{DC}$ & 0 & 0 & $*$ & + & + & + & 0 & + & $v_{1,1}$ \\
\hline$W B_{2}$ & $\mathrm{NTPPi}$ & & & & & & & & & $v_{1,2}$ \\
\hline$W N$ & $\mathrm{DC}$ & 0 & 0 & $*$ & - & - & - & - & 0 & $v_{1,1}$ \\
\hline$W N$ & $\mathrm{DC}$ & 0 & $*$ & + & + & $*$ & 0 & 0 & 0 & $v_{1,2}$ \\
\hline$R Y$ & $\emptyset$ & & & & & & & & & \\
\hline$R G_{1}$ & $\mathrm{NTPP}$ & & & & & & & & & $v_{1,1}$ \\
\hline$\cdots$ & $\cdots$ & $\cdots$ & $\cdots$ & $\cdots$ & $\cdots$ & $\cdots$ & $\cdots$ & $\cdots$ & $\cdots$ & $\cdots$ \\
\hline$B_{2} N$ & $\mathrm{DC}$ & + & 0 & $*$ & - & - & - & 0 & 0 & $v_{1,1}$ \\
\hline$B_{2} N$ & $\mathrm{DC}$ & - & $*$ & + & + & $*$ & + & 0 & 0 & $v_{1,2}$ \\
\hline
\end{tabular}

occluded, the set of cocircuits can still be computed and therefore, the number of correspondences with the views of the database can still be calculated. In this case, obviously, its selectivity decreases.

\section{Experimental Results}

The method has been fully implemented and experiments with different sets of $3 \mathrm{D}$ objects have been carried out to validate it. Sixteen views of each object with angular separation of 22.5 degrees have been used for the experiments. These images have been segmented using the segmentation method described in [11]. Then, the index of the learning set of eight views per object taken at the angles $0,45,90,135,180,225,270$ and 315 has been created. In the recognition process the set of cocircuits of each image of the test set composed by the eight views not used in the learning process that is, the views taken at angles: 22.5, 67.5, 115.5, 157.5, 202.5, 247.5, 292.5 and 337.5 degrees, has been calculated. The experimental results are encouraging and currently we are refining the method introducing a distance measure between set of cocircuits.

\section{Conclusions}

In this paper a new method for indexing a database of views of 3D object has been presented. It is based on the combination of two qualitative representations derived from the region connection calculus and oriented matroid theory. This combination of qualitative representations characterizes the local and global topology of the regions of an image, is invariant under affine and Euclidean transformation of the views, intrinsically robust to discretization errors of the image and insensitive to small displacements of the point of view. 


\section{References}

1. Serratosa, F., Alquézar, R., Sanfeliu, A.: Function-described for modeling objects represented by attributed graphs. Pattern Recognition 36 (2003) 781-798

2. Cohn, A., Bennett, B., Gooday, J., Gotts, N.M.: Qualitative spatial representation and reasoning with the region connection calculus. GeoInformatica 1 (1997) 275316

3. Björner, A., Vergnas, M.L., Sturmfels, B., White, N., Ziegler, G.M.: Oriented Matroids. Volume 43 of Encyclopedia of Mathematics and its Applications. Cambridge University Press (1993)

4. Lamdan, Y., Schwartz, J.T., Wolfson, H.J.: Affine invariant model-based object recognition. IEEE Transactions on Robotics and Automation 6 (1990)

5. Carlsson, S.: Combinatorial geometry for shape representation and indexing. In: Proceedings of the International Workshop on Object Representation for Computer Vision. (1996)

6. Egenhofer, M.J., Franzosa, R.D.: Point set topological relations. International Journal of Geographical Information Systems 5 (1991) 161-174

7. Bokowski, J., Sturmfels, B.: Computational Synthetic Geometry. Volume 1355 of Lecture Notes in Mathematics. Springer-Verlag (1989)

8. Richter-Gebert, J., Ziegler, G.M.: Oriented matroids. In Goodman, J.E., O’Rourke, J., eds.: Handbook of Discrete and Computational Geometry. CRC Press (1997)

9. Rourke, J.O.: Computational Geometry in C. Cambridge University Press (1999)

10. Toussaint, G.T.: Solving geometric problems with the rotating calipers. In: Proceedings of IEEE MELECON'83, Athens, Greece (1983)

11. Comaniciu, D., Meer, P.: Mean shift: A robust approach toward feature space analysis. IEEE Transactions on Pattern Analysis and Machine Intelligence 24 (2002) 603-619 